\title{
PARAMAGNETIC STATE OF HYDROGEN-PASSIVATED DOUBLE-DONOR CENTERS IN SILICON*
}

\section{T. Gregorkiewicz, I.S. Zevenbergen, Yu.V. Martynov AND C.A.J. AMmerlaAN}

Van der Waals-Zeeman Laboratorium, Universiteit van Amsterdam Valckenierstraat 65-67, 1018 XE Amsterdam, The Netherlands

Hydrogenation of two double donor centers in silicon - substitutional sulfur and thermal donor - is studied by electron paramagnetic resonance and electron-nuclear double resonance. For both centers the existence of a new paramagnetic $S=1 / 2$ state identified as the neutral charge state of the double donor passivated with a single hydrogen atom is concluded. The microscopic structure of such complexes is discussed.

PACS numbers: 76.30.Lh, 61.72. Bb, 76.70.Dx

\section{Introduction}

Recently hydrogen has been recognized as one of the major contaminants of high-grade commercial silicon. It is easily introduced in a variety of ways, which include some standard techniques as used in silicon device processing, and can easily migrate through a crystal even at moderate temperatures. Upon interaction with atomic hydrogen the electrical activity of all kinds of impurities (donor or acceptor, shallow or deep) can be terminated. The passivation mechanism can be monitored by various experimental techniques; in this paper we report on EPR and ENDOR investigations of two double donor centers in silicon: substitutional sulfur and thermal donors (TD's).

In case of a single donor, which is paramagnetic in its neutral charge state, the paramagnetism is lost by passivation. Consequently magnetic resonance cannot be applied in this case. A double donor, which is characterized by three different charge states within the band gap, may be passivated with one or two hydrogen atoms. This may create the situation when a passivated center becomes accessible to magnetic resonance spectroscopy, since a singly passivated double donor in its neutral charge state would be paramagnetic with spin $S=1 / 2$.

*This project has been partially sponsored by the Foundation for Fundamental Research on Matter [Stichling voor Fundamenteel Onderzoek der Materie (FOM)]. 


\section{Sulfur-hydrogen complexes}

In the past isolated S and S-S pairs were identified by EPR/ENDOR [1] spectroscopy. Upon exposure to hydrogen plasma passivation of their electrical activity has been concluded [2]. The kinetics of this process was shown to involve a single step mechanism, consistent with the participation of a single hydrogen atom. In line with the introductory remark such a process should lead to a simultaneous generation of new single donor center. More recently such a center was indeed detected in IR absorption by Peale et al. [3].

In the current study we have used $n$-type, phosphorus-doped silicon diffused with sulfur $\left(1370^{\circ} \mathrm{C}, 48 \mathrm{~h}\right)$ and subsequently with hydrogen or deuterium $\left(1250^{\circ} \mathrm{C}\right.$, $1 / 2 \mathrm{~h}$ ). The EPR measurements on thus prepared samples revealed the presence of two new EPR spectra of $\langle 111\rangle$ axial symmetry with a somewhat different degree of anisotropy [4]. These, labeled Si-NL54 and Si-NL55, were partly overlapping and could be resolved only by the field-scanned ENDOR (FSE) technique. The observed patterns showed splitting in two components being thus indicative of the hyperfine interaction with hydrogen. This was confirmed by ENDOR measurements where spectra symmetric with respect to the Zeeman frequency of a free proton have been observed. The involvement of a single hydrogen atom in each of the newly reported centers is also consistent with the measurements on samples doped with deuterium. In order to conclusively establish the participation of sul-

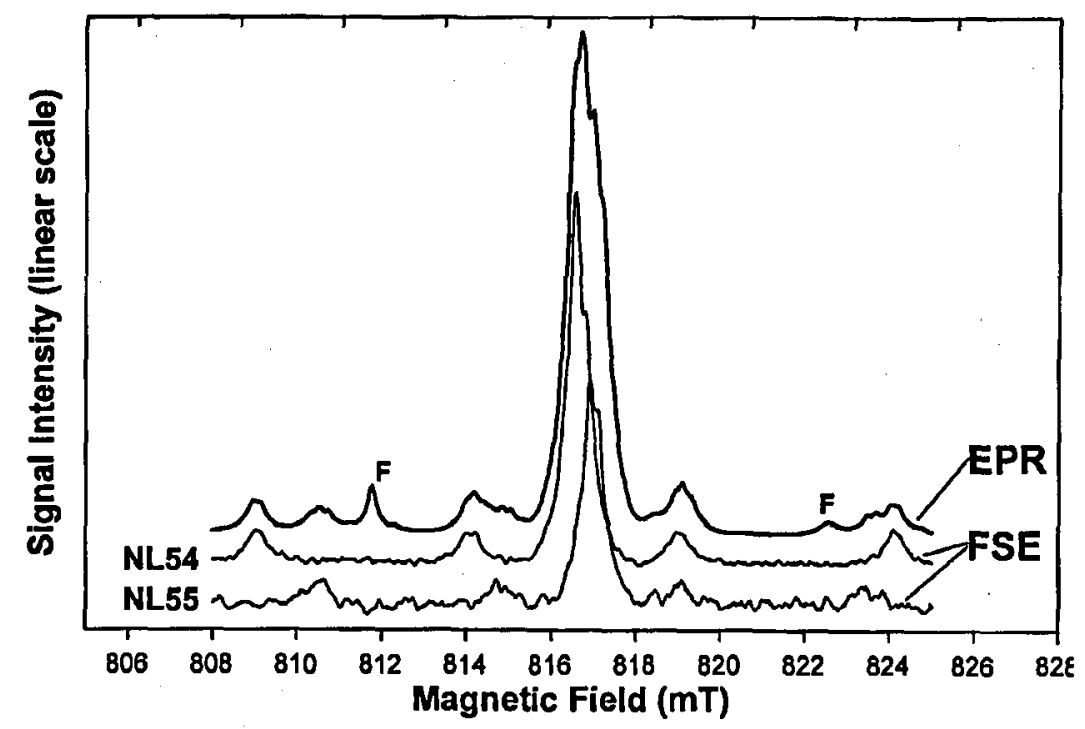

Fig. 1. The EPR and FSE spectra of Si-NL54 and Si-NL55 centers for magnetic field $\boldsymbol{B} \|\langle 100\rangle$ in the sample diffused with hydrogen and isotopically enriched sulfur. To each central line $(I=0)$ a fourfold spectrum $(I=3 / 2)$ belongs showing the involvement of a single ${ }^{33} \mathrm{~S}$ nucleus (the lines labelled $F$ belong to a different spectrum). The spectra were recorded at a microwave frequency of $\nu=22.8637 \mathrm{GHz}$ and at a temperature of $9 \mathrm{~K}$. 
fur in the Si-NL54 and Si-NL55 centers samples doped with isotopically enriched sulfur $\left(25 \%{ }^{33} \mathrm{~S}, I=3 / 2\right)$ were prepared. The EPR spectra recorded for these samples displayed a clearly resolved hyperfine interaction with one sulfur nucleus and identical hydrogen ENDOR. The EPR- and FSE-recorded spectra are compared in Fig. 1.

Based on the current results we identify the Si-NL54 and Si-NL55 centers as two configurations of a sulfur-hydrogen pair, i.e. a substitutional sulfur (double) donor passivated by one hydrogen atom. Consistent with the $S=1 / 2$ spin value we assign the spectra to the neutral charge state of such complexes. Such an identification is further supported by IR absorption measurements which reveal the presence of the characteristic $1 s \leftrightarrow 2 p$ electronic transitions assigned earlier to the S-II donor [3]. The proposed substitutional position of sulfur follows from the studies on isolated sulfur. Following the experimental findings the position of the hydrogen atom has to be along a $\langle 111\rangle$ direction with respect to sulfur, i.e., bond-centered (BC), or one of two antibonding (AB) sites on $S$ and nearest-neighbor $\mathrm{Si}$. We note here that our results do not support the conclusions of the theoretical study by Yapsir et al. [5] who predicted a distorted BC site for hydrogen in the S-II complex. On the other hand, they are consistent with the first-order kinetics of hydrogen passivation.

\section{Thermal donor-hydrogen complexes}

TD's are certainly among the most complicated defect centers in silicon and their microscopic picture is still missing. In EPR Si-NL8 and Si-NL10 spectra, both of orthorhombic-I symmetry, are now associated with TD's. One of them, Si-NL8, has been identified as a singly ionized state $\mathrm{TD}^{+}$; the microscopic identification of the second remains controversial. Upon exposure to hydrogen two effects have been reported: passivation [6], and enhanced TD formation [7]. A possible explanation of the latter phenomenon was offered by Estreicher [8] in a form of hydrogen-enhanced oxygen diffusion. The proposed mechanism could lead to a situation in which the formation of a TD center and its passivation are accomplished in a single step.

Being aware of the importance of hydrogen influence we undertook to investigate a possible involvement of hydrogen in the structure of the Si-NL10 center. In a sample with a strong Si-NL10 signal but not intentionally doped with hydrogen we have indeed detected a new ENDOR spectrum [9], which appeared to be symmetric with respect to the Zeeman frequency of a free proton - see Fig. 2. By recording the ENDOR spectra for different resonance field values and monitoring the frequency shift we unambiguously identified hydrogen as being responsible for the detected hyperfine interaction. IIaving established that, we intentionally doped a series of samples with hydrogen. In all of these hydrogen ENDOR spectra similar to that depicted in Fig. 2 were observed.

The hydrogen ENDOR spectrum was found to be a superposition of independent components, each of them being related to a different Si-NL10 species. From the angular dependence of the hydrogen ENDOR the symmetry of the defect was established as triclinic. Further, participation of a single $\mathrm{H}$ atom per Si-NL10 species has been concluded. The hyperfine interaction was found to be very small explaining its absence in an EPR spectrum. The spin localization on the hydrogen 


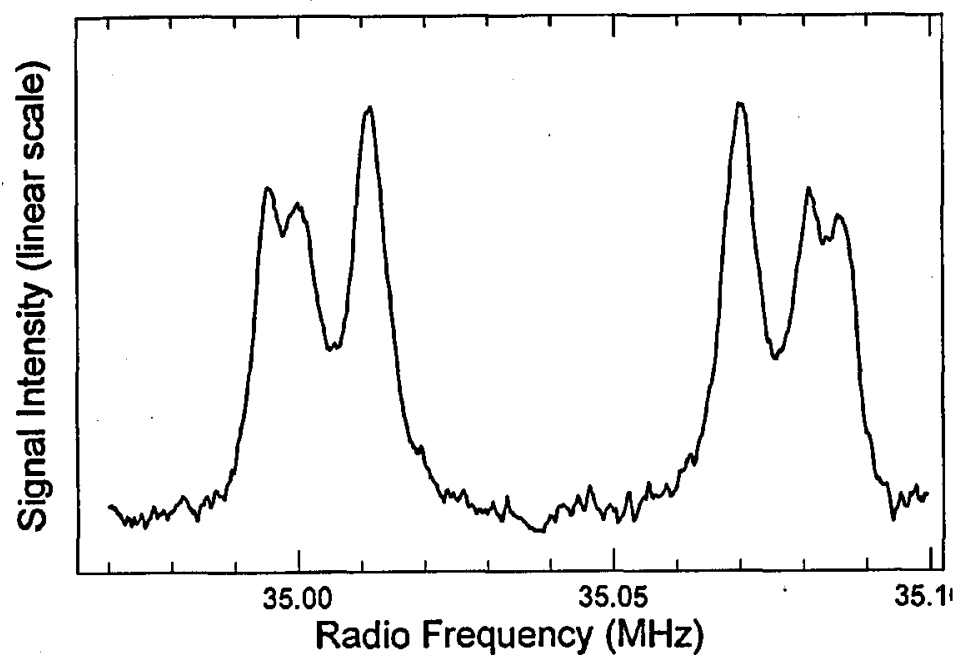

Fig. 2. The hydrogen ENDOR spectrum observed in a Cz-Si:P sample after $470^{\circ} \mathrm{C}$, $55 \mathrm{~h}$ heat treatment, recorded with $B \|\langle 111\rangle$ direction. The free-proton resonance frequency is at about $35.04 \mathrm{MHz}$.

nucleus was estimated to be (very) small, but nevertheless comparable to that found earlier for oxygen. The current findings allow us to identify the Si-NL10 center as the neutral charge state of a singly passivated TD, consistent also with all other characteristic features of the Si-NL10 structure and its similarities with the Si-NL8.

\section{Conclusions}

We have shown that hydrogenation of monocrystalline silicon doped with double donors may lead to the formation of double donor-hydrogen pairs which have single donor character and are therefore paramagnetic in their neutral charge state.

\section{References}

[1] A.B. van Oosten, C.A.J. Ammerlaan, Phys. Rev. B 38, 13291 (1988).

[2] G. Pensl, G. Roos, C. Holm, E. Sirtl, N.M. Johnson, Appl. Phys. Lett. 51, 451 (1987).

[3] R.E. Peale, K. Muro, A.J. Sievers, Mater. Sci. Forum 65-66, 151 (1990).

[4] I.S. Zevenbergen, T. Gregorkiewicz, C.A.J. Ammerlaan, to appear in Phys. Rev. B, 1995.

[5] A.S. Yapsir, P. Deák, Raj K. Singh, L.C. Snyder, J.W. Corbett, T.-M. Lu, Phys. Rev. B 38, 9936 (1988).

[6] N.M. Johnson, S.K. Hahn, H.J. Stein, Mater. Sci. Forum 10-12, 585 (1986).

[7] R. Murray, A.R. Brown, R.C. Newman, Mater. Sci. Eng. B 4, 299 (1989).

[8] S.K. Estreicher, Phys. Rev. B 41, 9886 (1990).

[9] Yu.V. Martynov, T. Gregorkiewicz, C.A.J. Ammerlaan, Phys. Rev. Lett. 74, 2030 (1995). 